\title{
Stochastic Modeling of Coherent Phenomena in Strongly Inhomogeneous Media
}

\author{
V. L. Kuz'min*,a ${ }^{\text {, I. V. Meglinski }}{ }^{\text {b,c }}$, and D. Y. Churmakov ${ }^{c}$ \\ ${ }^{a}$ St. Petersburg Institute of Trade and Economics, ul. Novorossiǔskaya 50, St. Petersburg, 194021 Russia \\ *e-mail: Vladimir.Kuzmin@paloma.spbu.ru \\ ${ }^{b}$ Saratov State University, ul. Universitetskaya 42, Saratov, 410026 Russia \\ ${ }^{c}$ Cranfield University, School of Engineering, Cranfield, MK43 OAL, UK
}

\begin{abstract}
A procedure of numerical simulation for coherent phenomena in multiply scattering media is developed on the basis of the juxtaposition of a Monte Carlo stochastic method with an iterative approach to the solution of the Bethe-Salpeter equation. The time correlation function and the interference component of coherent backscattering are calculated for scalar and electromagnetic fields. The results of simulation are in good agreement with experimental results, as well as with theoretical results obtained by generalizing the Milne solution.
\end{abstract}

\section{INTRODUCTION}

Stochastic modeling within a Monte Carlo method [1-10] has recently found wide application in the study of coherent phenomena associated with the propagation of light in randomly inhomogeneous media. These phenomena (coherent backscattering, spatial and time correlations of intensity) are attributed to the wave nature of light and, in spite of the multiple scattering regime, are observed in a wide variety of dielectric systems [11], such as colloidal suspensions, liquid crystals, and biological tissues.

The standard modeling technique for radiation in a random medium is based on the concept of intensity transfer. The phase relations between the fields that contribute to the intensity remain outside the framework of this modeling; one needs a special approach to take into account these relations.

The theory of multiple scattering in random media, including the theory of coherent and interference phenomena, is based on the Bethe-Salpeter equation (see [12]). In the present paper, we juxtapose a stochastic Monte Carlo method [13] with a theoretic method that is based on the representation of the solution to the Bethe-Salpeter equation as a series in scattering orders to demonstrate how the standard Monte Carlo method is generalized to a unified approach to the numerical simulation of the time correlation function of intensity, coherent backscattering, and other coherent phenomena that require the consideration of phase shifts.

Due to the multiple scattering, polarized light is completely depolarized; this fact allows one to restrict the consideration to a scalar field. However, in the case of backscattering, the scattered light remains partially polarized due to the contributions of lower order scat- tering terms. Experiments point to a significant role of polarization in backscattering [14-16].

In [17-22], the problem of multiple scattering by point, Rayleigh, particles was solved by generalizing the Milne solution to the case of electromagnetic waves. In [17. 18], a vector transport equation was solved for strictly backward scattering with regard to the interference component, and, in [19,20], the angular dependence of the backscattering peak was calculated with regard to polarization. In [21], a solution for the time correlation function was obtained. In [22], this solution was generalized to the case of finite-size scatterers.

In [2], a Monte Carlo procedure was applied to the calculation of the intensity of coherent backscattering, including a coherent component; for Rayleigh scattering, the peak of coherent backscattering proved to be much less than the enhancement predicted by the exact solution [19, 22]. In [23], the authors also simulated backscattering of electromagnetic waves; they calculated the rate of depolarization of linearly polarized light as a function of the number of scattering events. For Rayleigh scattering, the function obtained coincides with that predicted in [24], which was obtained within a diffusive approximation; however, as the anisotropy of the single-scattering cross section increases, the result of numerical simulation appreciably differs from theoretical results.

In the present paper, we develop a method for stochastic modeling of coherent phenomena with regard to the polarization of an electromagnetic field. For Rayleigh scattering, the numerical results obtained are in agreement with the theoretical results. This fact suggests that the data obtained in the general case of anisotropic scattering cross section are reliable. 
We consider the case, most frequently used in theoretical investigations, of the scattering of radiation in a medium that occupies a half-space with a plane boundary. To facilitate the account of the method, we consider the normal incidence and scattering close to the backward scattering.

In Section 2, we present general expressions for the time correlation function and the interference component of backscattering. In Section 3, we compare the methods of summation of ladder diagrams with a Monte Carlo method and present the results of simulation for the time correlation function and coherent backscattering for a scalar field. In Section 4, we consider the results of simulation for linearly polarized light. In the Conclusion, we discuss the results.

\section{TRANSFER OF FIELD CORRELATIONS}

Suppose that a medium occupies the half-space $z>$ 0 , where $z$ is the Cartesian coordinate normal to the boundary of the medium and $\mathbf{k}_{\mathrm{i}}$ and $\mathbf{k}_{\mathrm{s}}$ are the wave vectors of the incident and scattered plane waves.

The transfer of field correlations in an inhomogeneous dispersion medium with random space-time fluctuations of dielectric constant is described by the Bethe-Salpeter integral equation

$$
\begin{gathered}
\hat{\Gamma}\left(\mathbf{R}_{2}, \mathbf{R}_{1} t \mid \mathbf{k}_{s}, \mathbf{k}_{i}\right)=k_{0}^{4} \tilde{G}\left(\mathbf{k}_{\mathrm{s}}-\mathbf{k}_{\mathrm{i}}, t\right) \delta\left(\mathbf{R}_{2}-\mathbf{R}_{1}\right) \hat{I} \\
+k_{0}^{4} \int d \mathbf{R}_{3} \tilde{G}\left(\mathbf{k}_{\mathrm{s}}-\mathbf{k}_{23}, t\right) \hat{\Lambda}\left(\mathbf{R}_{2}-\mathbf{R}_{3}\right) \\
\times \hat{\Gamma}\left(\mathbf{R}_{3}, \mathbf{R}_{1}, t \mid \mathbf{k}_{23}, \mathbf{k}_{\mathrm{i}}\right) .
\end{gathered}
$$

Here, $\Gamma_{\beta_{1} \beta_{2} \alpha_{1} \alpha_{2}}\left(\mathbf{R}_{2}, \mathbf{R}_{1}, t \mid \mathbf{k}_{\mathrm{s}}, \mathbf{k}_{\mathrm{i}}\right)$ is the propagator, or the Green's function of the Bethe-Salpeter equation, which represents a fourth-rank tensor. It describes the transfer of two complex-conjugate fields that arrive at the point $\mathbf{R}_{1}$ with a time shift $t$, the wave vectors $\mathbf{k}_{\mathrm{i}}$, and the polarizations described by the Cartesian indices $\alpha_{1}$ and $\alpha_{2}$, and go out from the point $\mathbf{R}_{2}$ with the wave vectors $\mathbf{k}_{\mathrm{s}}$ and the polarizations described by the Cartesian indices $\beta_{1}$ and $\beta_{2}$. Here, $k_{0}=2 \pi / \lambda$ is the wavenumber; $\lambda$ is the wavelength; $k_{\mathrm{s}}=k_{\mathrm{i}}=k=n k_{0} ; n$ is the refractive index of the random medium: $n=n_{1}+i n_{2}$, where $n_{1}$ and $n_{2}$ are the real and imaginary parts of $n$, respectively; the imaginary part $n_{2}$ defines the photon mean free path $\left(2 n_{2} k_{0}\right)^{-1}=l$. The quantity

$$
\mathbf{k}_{i j}=k\left(\mathbf{R}_{i}-\mathbf{R}_{j}\right)\left|\mathbf{R}_{i}-\mathbf{R}_{j}\right|^{-1}
$$

defines the wave vector between the $i$ th and $j$ th scattering events. The fourth-rank tensor $\hat{\Lambda}(\mathbf{R})$,

$$
\begin{aligned}
& \Lambda_{\alpha \beta \mu v}(\mathbf{R})=\left(\hat{I}-\frac{\mathbf{R} \otimes \mathbf{R}}{R^{2}}\right)_{\alpha \mu} \\
& \times\left(\hat{I}-\frac{\mathbf{R} \otimes \mathbf{R}}{R^{2}}\right)_{\beta v} \frac{\exp (-R / l)}{R^{2}},
\end{aligned}
$$

represents a direct product of the complex-conjugate pair of Green's functions of the Maxwell wave equation in the far-field region and describes the transformation of a pair of fields with polarizations $\mu$ and $\nu$ into a pair of fields with polarizations $\alpha$ and $\beta$ in a single scattering event.

In the weak scattering approximation $(\lambda \ll l)$, which is usually valid in the dielectric systems under consideration, $\tilde{G}(\mathbf{q}, t)$ represents the Fourier image of the correlation function of space-time fluctuations of the dielectric constant:

$$
\begin{aligned}
\tilde{G}(\mathbf{q}, t)= & \frac{1}{(4 \pi)^{2}} \int d \mathbf{r}\langle\delta \varepsilon(0,0) \delta \varepsilon(\mathbf{r}, t)\rangle \\
& \times \exp (-i \mathbf{q} \cdot \mathbf{r}) .
\end{aligned}
$$

A key role in the problems of multiple scattering is played by the optical theorem, which relates the singlescattering cross section to the scattering length $l_{\mathrm{s}}$. For an electromagnetic field, the optical theorem in the approximation of weak scattering, or the Born approximation, is expressed as

$$
l_{\mathrm{s}}^{-1}=\Gamma_{\mathrm{R}}^{-1} k_{0}^{4} \int d \Omega_{\mathrm{s}} \tilde{G}_{0}\left(\mathbf{k}_{\mathrm{s}}-\mathbf{k}_{\mathrm{i}}\right) .
$$

Here, $\tilde{G}_{0}(\mathbf{q})=\tilde{G}(\mathbf{q}, t)$ is the Fourier image of the static correlation function of fluctuations of the dielectric constant, $\Gamma_{\mathrm{R}}=2\left(1+\overline{\cos ^{2} \theta}\right)^{-1}$ is the Rayleigh factor, and

$$
\overline{\cos ^{2} \theta}=\frac{\int d \Omega_{\mathrm{s}} \tilde{G}_{0}\left(\mathbf{k}_{\mathrm{s}}-\mathbf{k}_{\mathrm{i}}\right) \cos ^{2} \theta_{\mathrm{s}}}{\int d \Omega_{\mathrm{s}} \tilde{G}_{0}\left(\mathbf{k}_{\mathrm{s}}-\mathbf{k}_{\mathrm{i}}\right)}
$$

is the squared cosine, averaged over the single-scattering cross section, of the scattering angle between the wave vectors $\mathbf{k}_{\mathrm{i}}$ and $\mathbf{k}_{\mathrm{s}}$.

The photon mean free path $l$ and the scattering length $l_{\mathrm{s}}$ are related by the formula

$$
\frac{1}{l}=\frac{1}{l_{\mathrm{s}}}+\frac{1}{l_{\mathrm{a}}},
$$

where $l_{\mathrm{a}}$ is the characteristic length of the absorption due to inelastic scattering. For the media considered here, $l_{\mathrm{a}} \gg l$ and the ratio $l / l_{\mathrm{s}}$ is close to unity.

Let us define a normalized correlation function of fluctuations of the dielectric constant:

$$
p\left(\mathbf{k}_{\mathrm{i}}-\mathbf{k}_{\mathrm{s}}, t\right)=\frac{\tilde{G}\left(\mathbf{k}_{\mathrm{i}}-\mathbf{k}_{\mathrm{s}}, t\right)}{\int \tilde{G}\left(\mathbf{k}_{\mathrm{i}}-\mathbf{k}_{\mathrm{s}}, 0\right) d \Omega_{\mathrm{s}}} .
$$

For $t=0$, this function coincides with the phase function $p_{0}\left(\mathbf{k}_{\mathrm{i}}-\mathbf{k}_{\mathrm{s}}\right)=p\left(\mathbf{k}_{\mathrm{i}}-\mathbf{k}_{\mathrm{s}}, 0\right)$, which describes the cross section of single scattering. 
Iterating the Bethe-Salpeter equation and applying the optical theorem, we obtain the series

$$
\begin{gathered}
\hat{\Gamma}\left(\mathbf{R}_{2}, \mathbf{R}_{1}, t \mid \mathbf{k}_{s}, \mathbf{k}_{i}\right)=\Gamma_{\mathrm{R}} l_{\mathrm{s}}^{-1} p\left(\mathbf{k}_{\mathrm{i}}-\mathbf{k}_{\mathrm{s}}, t\right) \delta\left(\mathbf{R}_{2}-\mathbf{R}_{1}\right) \\
+\Gamma_{\mathrm{R}}^{2} l_{\mathrm{s}}^{-2} p\left(\mathbf{k}_{\mathrm{s}}-\mathbf{k}_{21}, t\right) \hat{\Lambda}\left(\mathbf{R}_{21}\right) p\left(\mathbf{k}_{21}-\mathbf{k}_{\mathrm{i}}, t\right) \\
+\Gamma_{\mathrm{R}}^{3} l_{\mathrm{s}}^{-3} \int d \mathbf{R}_{3} p\left(\mathbf{k}_{\mathrm{s}}-\mathbf{k}_{23}, t\right) \hat{\Lambda}\left(\mathbf{R}_{23}\right) p\left(\mathbf{k}_{23}-\mathbf{k}_{31}, t\right) \\
\times \hat{\Lambda}\left(\mathbf{R}_{31}\right) p\left(\mathbf{k}_{31}-\mathbf{k}_{\mathrm{i}}, t\right)+\ldots
\end{gathered}
$$

which is usually represented as a series of ladder diagrams.

Let us define a binary correlation function of a field that is observed at a large distance $r$ from the scattering medium as

$$
\begin{gathered}
\hat{C}^{(\mathrm{E})}\left(t \mid \mathbf{k}_{\mathrm{s}}, \mathbf{k}_{\mathrm{i}}\right) \\
=\hat{C}^{(L)}\left(t \mid \mathbf{k}_{\mathrm{s}}, \mathbf{k}_{\mathrm{i}}\right)+\hat{C}^{(\mathrm{V})}\left(t \mid \mathbf{k}_{\mathrm{s}}, \mathbf{k}_{\mathrm{i}}\right),
\end{gathered}
$$

where $\hat{C}^{(\mathrm{L})}\left(t \mid \mathbf{k}_{\mathrm{s}}, \mathbf{k}_{\mathrm{i}}\right)$ is a contribution of ladder diagrams that describes a noncoherent component, while $\hat{C}^{(\mathrm{V})}\left(t \mid \mathbf{k}_{\mathrm{s}}, \mathbf{k}_{\mathrm{i}}\right)$ is the interference component that is observed in the backscattered wave.

In the case of normal incidence and scattering close to the backward scattering, the ladder and interference components of the time correlation function of a field are given by

$$
\begin{gathered}
C_{\beta_{1} \beta_{2} \alpha_{1} \alpha_{2}}^{(\mathrm{L})}\left(t \mid \mathbf{k}_{\mathrm{s}}, \mathbf{k}_{\mathrm{i}}\right) \\
=\int d \mathbf{R}_{1} d \mathbf{R}_{2} \Gamma_{\beta_{1} \beta_{2} \alpha_{1} \alpha_{2}}\left(\mathbf{R}_{2}, \mathbf{R}_{1}, t \mid \mathbf{k}_{\mathrm{s}}, \mathbf{k}_{\mathrm{i}}\right) \\
\times \exp \left(-\frac{z_{1}+z_{2}}{l}\right)
\end{gathered}
$$

and

$$
\begin{gathered}
C_{\beta_{1} \beta_{2} \alpha_{1} \alpha_{2}}^{(\mathrm{V})}\left(t \mid \mathbf{k}_{s}, \mathbf{k}_{i}\right)=\int d \mathbf{R}_{1} d \mathbf{R}_{2} \\
\times\left[\Gamma_{\beta_{1} \alpha_{2} \alpha_{1} \beta_{2}}\left(\mathbf{R}_{2}, \mathbf{R}_{1}, t \mid \frac{\mathbf{k}_{\mathrm{s}}-\mathbf{k}_{\mathrm{i}}}{2}, \frac{\mathbf{k}_{\mathrm{i}}-\mathbf{k}_{\mathrm{s}}}{2}\right)\right. \\
\left.-k_{0}^{4} \tilde{G}\left(\mathbf{k}_{\mathrm{s}}-\mathbf{k}_{\mathrm{i}}, t\right) \delta\left(\mathbf{R}_{2}-\mathbf{R}_{1}\right) \delta_{\alpha_{1} \beta_{1}} \delta_{\alpha_{2} \beta_{2}}\right] \\
\times \exp \left(-\frac{z_{1}+z_{2}}{l}+i\left(\mathbf{k}_{\mathrm{s}}+\mathbf{k}_{\mathrm{i}}\right)_{\perp} \cdot\left(\mathbf{R}_{2}-\mathbf{R}_{1}\right)_{\perp}\right),
\end{gathered}
$$

respectively, where the subscript " $\perp$ " denotes the component perpendicular to the boundary of the medium. It is easily seen that, for backward scattering, when $\mathbf{k}_{\mathrm{s}}=$ $-\mathbf{k}_{\mathrm{i}}$, the polarized component of the interference contribution $\hat{C}^{(\mathrm{V})}\left(t \mid \mathbf{k}_{\mathrm{s}}, \mathbf{k}_{\mathrm{i}}\right)$ coincides with the polarization component of the main, noncoherent, contribution $\hat{C}^{(\mathrm{L})}\left(t \mid \mathbf{k}_{\mathrm{s}}, \mathbf{k}_{\mathrm{i}}\right)$ before subtracting the contribution of single scattering, while the depolarized components do not coincide.

The noncoherent component defines the time correlation function of a field

$$
g_{1}(t)=\frac{C^{(\mathrm{L})}\left(t \mid-\mathbf{k}_{\mathrm{i}}, \mathbf{k}_{\mathrm{i}}\right)}{C^{(\mathrm{L})}\left(0 \mid-\mathbf{k}_{\mathrm{i}}, \mathbf{k}_{\mathrm{i}}\right)}
$$

Disregarding non-Gaussian long-range components, we can represent the intensity correlation function as a square of the field correlation function: $g_{2}(t)=1+$ $g_{1}^{2}(t)$.

For $t=0$, the interference component (2.10) describes the peak of coherent backscattering

$$
I^{\mathrm{CBS}}\left(\theta_{\mathrm{s}}\right)=\frac{C^{(\mathrm{V})}\left(0 \mid \mathbf{k}_{\mathrm{s}}, \mathbf{k}_{\mathrm{i}}\right)}{\left(0 \mid-\mathbf{k}_{\mathrm{i}}, \mathbf{k}_{\mathrm{i}}\right)}
$$

and its angular dependence.

\section{SIMULATION OF A SERIES IN SCATTERING ORDERS: A SCALAR FIELD}

Let us juxtapose the procedure of analytic summation of a series of ladder diagrams with a Monte Carlo method that combines the calculation scheme of stochastic trajectories with the application of statistical weights [26, 27]. First, consider a scalar field. When passing to the case of a scalar field, the tensor $\hat{\Lambda}(\mathbf{R})$ is replaced by the scalar function $\Lambda_{0}(R)=R^{-2} \exp (-R / l)$, and the Rayleigh factor $\Gamma_{\mathrm{R}}$ is replaced by unity. The first term of the iterative series describes single scattering, the second describes double scattering, etc.

A Monte Carlo method describes the radiation propagation as a random process that consists of one, two, $\ldots, n$ scattering events. The addition of one link of the ladder $\Lambda\left(R_{j j-1}\right) p_{0}\left(\mathbf{k}_{j}-\mathbf{k}_{j-1}\right)$ in the theoretical description is realized in the numerical experiment by a path $R$ traversed by a photon (a packet of photons) up to the next scattering event. The random photon mean free path $R$ between two successive scattering events is defined by the Poisson distribution [13]:

$$
f(R)=l^{-1} \exp (-R / l)
$$

This distribution implies that $R=-l \ln \xi$, where $\xi$ is the probability of the fact that the mean free path is greater than $R$; the value of $\xi$ is chosen by a random number generator from the interval $[0,1]$. The propagation 
direction of a photon packet in each event of elastic scattering is defined randomly with regard to the phase function (2.6). As a result, one obtains a stochastic trajectory of a photon that is emitted at the point $\mathbf{R}_{0}=\mathbf{R}_{S}$, experienced $n$ collisions at the points $\mathbf{R}_{1}, \ldots, \mathbf{R}_{n}$, and detected on the surface at the point $\mathbf{R}_{n+1}=\mathbf{R}_{\mathrm{D}}$; this trajectory randomly simulates the $n$ th-order term in the above-mentioned iterative series (Fig. 1).

Let $W_{i}$ be the statistical weight of the $i$ th photon that reaches a detector. Then, the sum of these weights defines, in arbitrary units, the intensity of scattered radiation at the detection point,

$$
I=\Sigma_{i} W_{i} .
$$

In the case of isotropic scattering, $W_{i}=1 / N_{\mathrm{ph}}$, where $N_{\mathrm{ph}}$ is the sample size; in the case of anisotropic scattering, this quantity is multiplied by a product of phase functions.

The complexity of analytic calculations is associated with the fact that the integrals over $\mathbf{R}_{i}$ cannot be uncoupled because the phase functions depend on the mutual disposition of scattering particles. The numerical simulation uncouples this chain and, at each step, randomly defines the direction and the magnitude of the mean free path of a photon packet.

By virtue of the normalization condition for the phase function,

$$
\int p_{0}\left(\mathbf{k}_{\mathrm{i}}-\mathbf{k}_{\mathrm{s}}\right) d \Omega=1,
$$

the statistical weight of a photon packet is not changed after each scattering event. In theoretical description, the conservation of the weight of a packet is fulfilled according to the optical theorem. Indeed, since

$$
\int \Lambda_{0}(R) d \mathbf{R}=4 \pi l,
$$

the expansion parameter of iterative series (2.7) is given by

$l_{\mathrm{s}}^{-1} \int d \Omega_{n} \int d \mathbf{R}_{i+1} \Lambda_{0}\left(\mathbf{R}_{i+1}-\mathbf{R}_{i}\right) p_{0}\left(\mathbf{k}_{i+1}-\mathbf{k}_{i}\right)=l_{\mathrm{s}}^{-1} l$.

Let us show that it is the form of the propagator $\Lambda_{0}(R)$ that leads to distribution (3.1). In the absence of absorption, the quantity $l_{\mathrm{s}}^{-1} l$ is exactly equal to unity, which points to the conservation of the weight of the photon packet. In analytic calculations, it is the condition $l_{\mathrm{s}}^{-1} l=1$ that makes the method of successive approximations inapplicable to solving the Bethe-Salpeter equation.

A photon contributes to the detected signal provided that it intersects the boundary of the medium at a given angle at the detection point. Then, the trajectory of the next photon is modeled. The sample size of incident

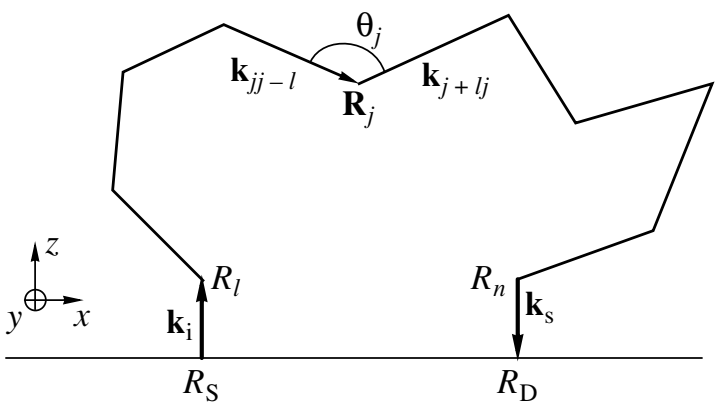

Fig. 1. Trajectory of random walks of a photon from the input point $\mathbf{R}_{\mathrm{S}}$ to the output point $\mathbf{R}_{\mathrm{D}} ; \mathbf{R}_{1}$ and $\mathbf{R}_{n}$ are the points of the first and the last, $n$ th, scattering events; $\mathbf{k}_{j j-1}$ and $\mathbf{k}_{j+1 j}$ are the wave vectors before and after the $j$ th scattering event; and $\theta_{j}$ is the angle between the above wave vectors.

photons varied from $10^{5}$ to $10^{7}$. The modeling of a photon trajectory terminated when the number of scattering events became greater than $10^{4}$. When the statistical weight of a photon became less than $10^{-3}$, we also ceased to follow up the trajectory of the photon. According to our estimates, the detection probability of such a photon on the surface is no greater than $10^{-2}$; therefore, our approach leads to error of at most $10^{-5}$. We controlled the accuracy of calculated parameters by the stability of their numerical values under increasing sample size. For a sample size of $10^{5}$, the intensity is stable up to at least four digits.

As the phase function, we used the Henyey-Greenstein function (see [28]). In [2, 29], the authors used the Rayleigh-Gans function.

When a fraction of scattered photons possessing the required properties (for example, phonons scattered into a narrow solid angle when modeling coherent backscattering) is small, the time it takes to accumulate reliable statistics may prove to be extremely large in the above-described standard modeling technique.

To improve the statistics, we applied a semianalytic method of modeling [30], which is also known as a method of local estimation [31], in which each photon contributes to the scattered radiation. Suppose that a trajectory contains $N$ scattering events. The weight of the $i$ th photon after $n(n<N)$ scattering events is $W_{n}^{(i)}$. The contribution of all trajectories with the number of scattering events $n$ is simulated by the term of iterative series (2.7) that describes $n$-tuple scattering in the ladder component (2.9):

$$
C^{(L)} \sim \sum_{i} W_{n}^{(i)} \exp \left(-z_{n}^{(i)} / l\right),
$$

where $z_{n}^{(i)}$ is the distance from the point of the last, $n$th scattering event to the boundary of the medium. Thus, in this approach, each photon contributes to the detected radiation. The accuracy of modeling is easily 


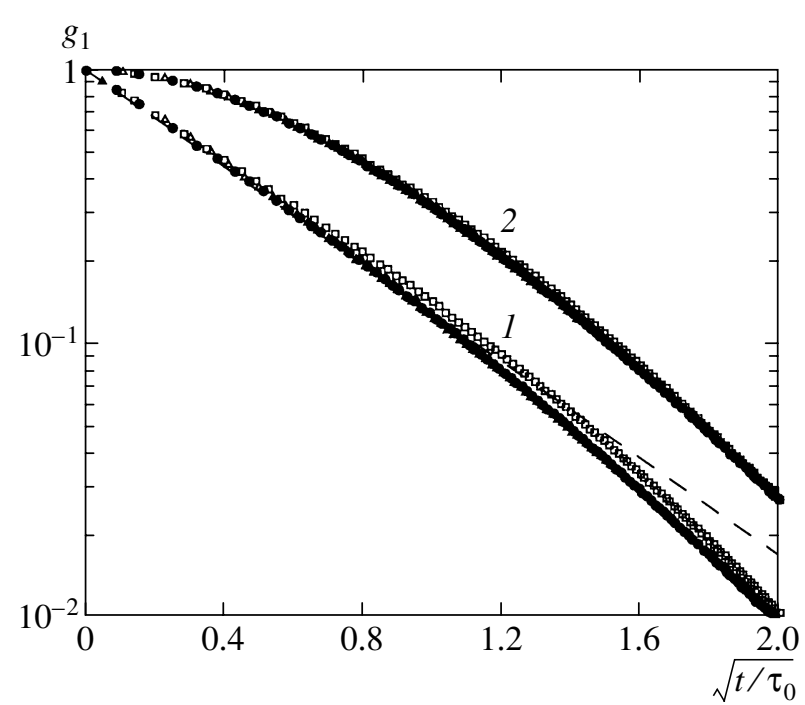

Fig. 2. The time correlation function of a field scattered backward by (1) a semi-infinite medium and (2) a layer of thickness $L=l^{*}$ as a function of the time argument $\sqrt{t / \tau}$ for three values of the anisotropy parameter $\overline{\cos \theta}=0(\square)$, $0.5(\bullet)$, and $0.9(\triangle)$; the dashed curve represents approximation by function (3.6) with $\gamma=2$.

monitored by comparing with analytic results, which can be obtained for lower orders of scattering. For example, in the case of isotropic scattering, the contributions of single and double scattering to the intensity are given by

$$
\begin{gathered}
I_{\text {single }}=l^{-1} \int d z_{1} d \mathbf{R}_{2} \delta\left(\mathbf{R}_{2}-\mathbf{R}_{1}\right) \exp \left(-\frac{z_{1}+z_{2}}{l}\right)=\frac{1}{2}, \\
I_{\text {double }}=(4 \pi)^{-1} l^{-2} \int d z_{1} \int d \mathbf{R}_{2} \Lambda_{0}\left(\mathbf{R}_{2}-\mathbf{R}_{1}\right) \\
\quad \times \exp \left(-\frac{z_{1}+z_{2}}{l}\right)=\ln \sqrt{2}=0.346 \ldots
\end{gathered}
$$

Using the method described, we reproduced these theoretical results by simulation with a high degree of accuracy.

The analysis involving the juxtaposition of the theoretical approach based on the Bethe-Salpeter equation with the Monte Carlo method allows us to generalize the latter method for modeling coherent multiple scattering phenomena.

The difference between the calculation of the time correlation function and the calculation of intensity lies in the fact that the direction of a scattered photon packet is determined by a generalized phase function $p\left(\mathbf{k}_{j}-\right.$ $\mathbf{k}_{j-1}, t$ ), which depends on the time shift $t$, rather than by the phase function itself. In the major part of known applications $[15,32]$, the authors investigate a diffusion mechanism of the time evolution of irregularities, when the time correlation function of the intensity fluctuations can be represented as a product of a static correlation function and an exponential function:

$$
p(q, t) \approx p_{0}(q) \exp \left(-D_{\mathrm{s}} q^{2} t\right)
$$

where $D_{\mathrm{s}}$ is the coefficient of self-diffusion. Thus, the time correlation function is calculated in the Monte Carlo method as

$$
g_{1}(t)=\sum_{i=1}^{N_{\mathrm{ph}}} W_{i} \exp \left(-2 \frac{t}{\tau} n_{i}\left(1-\frac{1}{n_{i}} \sum_{j}^{n_{i}} \cos \theta_{j}\right)\right),
$$

where $\tau=\left(D_{\mathrm{s}} k^{2}\right)^{-1}$ is the characteristic time of Brownian diffusion of a scatterer to a distance $\lambda$ and $\theta_{j}$ is the scattering angle in the $j$ th scattering event. The results remains virtually unchanged if we replace the sample average by the average over the phase function

$$
\frac{1}{n_{i}} \sum_{j}^{n_{i}} \cos \theta_{j} \longrightarrow \overline{\cos \theta}=\frac{\int d \Omega_{\mathrm{s}} \tilde{G}_{0}\left(\mathbf{k}_{\mathrm{s}}-\mathbf{k}_{\mathrm{i}}\right) \cos \theta_{\mathrm{s}}}{\int d \Omega_{\mathrm{s}} \tilde{G}_{0}\left(\mathbf{k}_{\mathrm{s}}-\mathbf{k}_{\mathrm{i}}\right)},
$$

in agreement with the diffusive approximation.

In the isotropic case, there is an exact Milne solution that allows one to control the results of simulation. The exact solution gives the following value for the ratio of the intensity of the total backscattered radiation to the intensity of single scattering: $I / I_{\text {single }}=8.455 \ldots$ (see [12]). The method of simulation described reproduces this value with an accuracy of at least four digits for a sample size on the order of $10^{5}$. To reduce the simulation time, we calculated, within the diffusive approximation, the contribution of photons for which the distance between the input and output points is several dozens of times greater than the mean free path $l$, whereas the contribution of photons that are emitted at a distance less than $l$ is calculated by the simulation scheme described.

Figure 2 represents the results of simulation for the time correlation function of field for three scattering media with different values of the anisotropy factor

$\overline{\cos \theta}=0,0.5$, and 0.9 . We chose the value $l=33 \mu \mathrm{m}$, which corresponds to the values of the transport length $l^{*}=l(1-\overline{\cos \theta})^{-1}$ ranging from $33 \mu \mathrm{m}$ for the isotropic case to $333 \mu \mathrm{m}$ for the case of strong anisotropy $(\overline{\cos \theta}=0.9)$. In terms of $\sqrt{t / \tau}$, the correlation function is practically universal and does not depend on the anisotropy of single scattering. Note also that these results are in good agreement with experimental data $[15,16]$. The obtained time correlation function of field is well described by the formula

$$
g_{1}(t) \propto \exp (-\gamma \sqrt{6 t / \tau}),
$$

which was proposed in [15]. 
The slope ratio $\gamma$ of the time correlation function of field in a medium with isotropic scattering cross section: Theoretical predictions and results of simulation

\begin{tabular}{l|l|c|c|c}
\hline Incident radiation & \multicolumn{1}{|c|}{ Scattered radiation } & Diffusive approximation & Milne solution & Monte Carlo method \\
\hline Plane wave & Plane wave & $2 \frac{\left(1+z^{*}\right)^{2}}{1+2 z^{*}}$ & $2[12,22]$ & $\approx 2$ \\
Plane wave & Point detector & $1+z^{*}$ & $\approx 1.53[22]$ & $\approx 1.53$ \\
Point source & Total scattered radiation & $z^{*}$ & & $\approx 1.2$ \\
\hline
\end{tabular}

Note: The Milne parameter is $z^{*} \approx 0.71$.

The deviation from a linear decay for very small times is attributed to insufficient statistics at large distances. As time increases, the contribution of long optical paths decreases, thus weakening the requirements on the statistical sample size.

Strictly speaking, the linear dependence on $\sqrt{t / \tau}$ is realized only for the scattering from a semi-infinite medium. In the case of scattering from a layer of finite thickness, the time correlation function decreases linearly with time $t$. However, even for layers with a thickness on the order of the transport mean free path, the time correlation function in terms of $\sqrt{t / \tau}$ weakly depends on the anisotropy parameter $\overline{\cos \theta}$.

The decay rate of the correlation function, which weakly depends on the specific character of a medium in dimensionless terms $t / \tau$, depends rather strongly on the geometry of the experiment. The table presents the values of the slope ratio $\gamma$ for isotropic scattering that are calculated in the diffusive approximation with the use of the Milne solution and obtained by simulation.

One can see that, in the case of a point source or a point detector, the decay rate of correlations is less than that in the case of plane waves. As pointed out in [15], this is associated with the fact that, in the case of plane waves, the relative contribution of longer optical paths increases and leads to a faster decay of the correlation function.

Note that, compared with the expression for the intensity of a noncoherent component, the expression for the intensity of the interference component of backscattering (see (2.10)) contains an additional factor $\exp \left(i \mathbf{q}_{\perp} \cdot\left(\mathbf{R}_{1}-\mathbf{R}_{2}\right)_{\perp}\right)$. Taking into account the translational invariance with respect to the coordinates $\mathbf{R}_{\perp}$, we can replace this factor by $\cos \left(\mathbf{q}_{\perp} \cdot\left(\mathbf{R}_{1}-\mathbf{R}_{2}\right)_{\perp}\right)$. When calculating the intensity of the coherent component of backscattering, we should multiply the weight of the $i$ th photon arriving at the interface with vector $\mathbf{k}_{\mathrm{s}}$ at distance $\left|\left(\mathbf{R}_{\mathrm{S}}-\mathbf{R}_{\mathrm{D}}^{(i)}\right)_{\perp}\right|$ from the input point $\mathbf{R}_{\mathrm{S}}$ by the factor $\cos \left(\mathbf{q}_{\perp}\left(\mathbf{R}_{\mathrm{S}}-\mathbf{R}_{\mathrm{D}}^{(i)}\right)_{\perp}\right)$ and sum over all photons. As a result, we obtain the following expression for the peak of coherent backscattering:

$$
I^{\mathrm{CBS}}\left(\theta_{\mathrm{s}}\right)=\sum_{i} W_{i} \cos \left(\mathbf{q}_{\perp} \cdot\left(\mathbf{R}_{\mathrm{S}}-\mathbf{R}_{\mathrm{D}}^{i}\right)_{\perp}\right)-I_{\text {single }} .
$$

The height, or enhancement, of the peak of backscattering is given by

$$
h^{\mathrm{CBS}}=\frac{2 I-I_{\text {single }}}{I},
$$

where $I$ is the intensity of the noncoherent component. We obtained $h^{\mathrm{CBS}}=1.87$ for isotropic scattering, which is in good agreement with the value $h_{\text {theor }}^{\mathrm{CBS}}=1.88$ [2] obtained on the basis of the Milne generalized solution. For $\overline{\cos \theta}=0.9$, we obtained $h^{\mathrm{CBS}}=1.99$, which also agrees with the expected theoretical value $h_{\text {theor }}^{\mathrm{CBS}}=2$ for $\overline{\cos \theta} \longrightarrow 1$.

Just as in the case of time correlations, the angular dependence of the peak of coherent backscattering calculated in the dimensionless variables $\tilde{q}=k l^{*} \sin \theta_{\mathrm{s}}$ is close to the universal one (Fig. 3) and is sufficiently well described by the formula

$$
I^{\mathrm{CBS}}\left(\theta_{\mathrm{s}}\right) \propto \exp \left(-\gamma k l^{*} \sin \theta_{\mathrm{s}}\right)
$$

with $\gamma=2$. Note that this dependence differs significantly from the dependence predicted by the diffusive approximation [24]:

$$
I_{\mathrm{diff}}^{\mathrm{CBS}} \propto 1-2 \frac{\left(l+z^{*} l^{*}\right)^{2}}{l+2 z^{*} l^{*}} k \sin \theta_{\mathrm{s}}
$$

for $k l^{*} \sin \theta_{\mathrm{s}} \ll 1$, where $z^{*}=0.71 \ldots$ is the Milne extrapolation parameter. The values of the slope ratio given by this formula for the isotropic scattering and the strongly isotropic scattering differ by a factor of three: $\gamma^{\text {(diff) }}=$ 2.3 for $\overline{\cos \theta}=0$, and $\gamma^{\text {(diff) }}=0.71$ for $\overline{\cos \theta} \longrightarrow 1$ (see [18]). Note that the results of simulation also show 


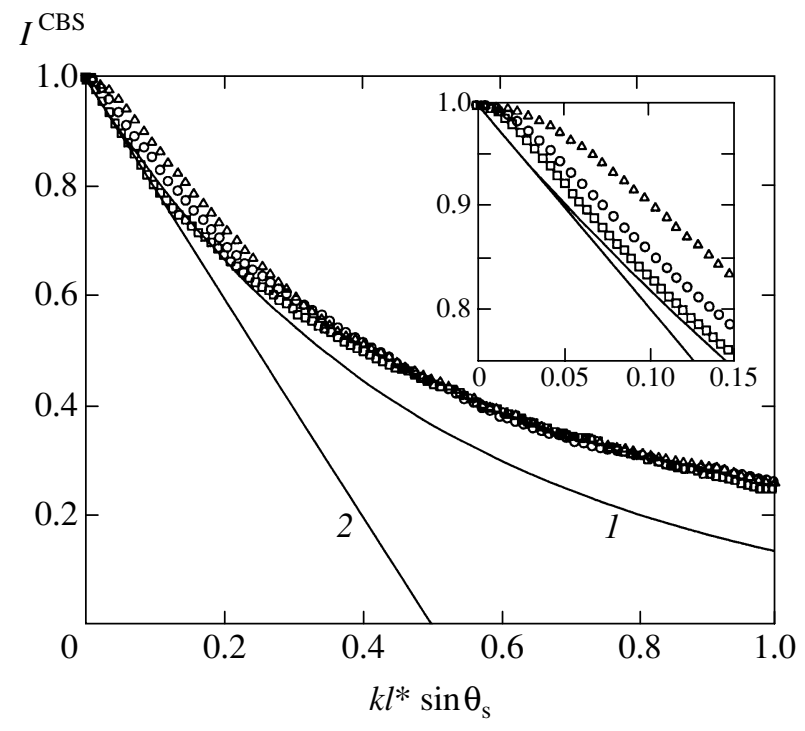

Fig. 3. Peak of coherent backscattering as a function of $k l^{*} \sin \theta_{\mathrm{s}} ; \lambda=0.6 \mu \mathrm{m} ; l=33 \mu \mathrm{m}$; and $\overline{\cos \theta}=0(\square), 0.5(\bigcirc)$, and $0.9(\triangle) ;(1)$ approximation by $\exp \left(-2 k l^{*} \sin \theta_{\mathrm{s}}\right)$ and (2) function of the form $1-2 k l^{*} \sin \theta_{\mathrm{s}}$. The inset shows the initial regions that demonstrate the deviation from universality.

that the decay rate of the peak of coherent backscattering decreases as the anisotropy parameter $\overline{\cos \theta}$ increases in the region of very small scattering angles, $k l^{*} \sin \theta_{\mathrm{s}} \leq 0.1$.

\section{CORRELATION EFFECTS FOR LINEARLY POLARIZED LIGHT}

In the case of an electromagnetic wave, one should additionally follow up the variation in the direction of the field, characterized by a polarization vector, along a random trajectory of a photon. According to (2.2), to this end, one should calculate the result of the action of the chain of operators [24]

$$
\prod_{j=1}^{n}\left(\hat{I}-\left(\mathbf{R}_{j+1}-\mathbf{R}_{j}\right) \otimes\left(\mathbf{R}_{j+1}-\mathbf{R}_{j}\right)\left|\mathbf{R}_{j+1}-\mathbf{R}_{j}\right|^{-2}\right)(
$$

on the incident field.

Suppose that, just as in the case of a scalar field, the weight of each incident photon is $1 / N_{\mathrm{ph}}$. In the electromagnetic field, one defines, in addition to the weight, the initial polarization of the photon field; in the general case, it is defined by three Cartesian coordinates. Suppose that the polarization of each incident photon is defined by a set of three numbers: $\mathbf{P}^{(i n)}=(1 ; 0 ; 0)$. This vector of initial polarization indicates that the incident field is polarized along the $x$ axis.

The polarization of a field is changed under scattering. In addition to the standard procedure of the sto- chastic determination of the direction of a photon after a collision and finding the weight function by the phase function, one should calculate a new vector of polarization $\mathbf{P}_{j+1}$ by the preceding vector $\mathbf{P}_{j}$ for each scattering event:

$$
\mathbf{P}_{j+1}=\left(\hat{I}-\left(\mathbf{R}_{j+1}-\mathbf{R}_{j}\right) \otimes\left(\mathbf{R}_{j+1}-\mathbf{R}_{j}\right)\left|\mathbf{R}_{j+1}-\mathbf{R}_{j}\right|^{-2}\right) \mathbf{P}_{j} .
$$

Suppose that a photon experiences $n$ scattering events. Then, after the last, $n$th scattering event, a photon arrives at the observation point $\mathbf{R}_{\mathrm{D}}$ with the polarization vector

$$
\begin{aligned}
& \mathbf{P}^{(\text {out })}=\prod_{j=1}^{n}\left(\hat{I}-\left(\mathbf{R}_{j+1}-\mathbf{R}_{j}\right) \otimes\left(\mathbf{R}_{j+1}-\mathbf{R}_{j}\right)\right. \\
&\left.\times\left|\mathbf{R}_{j+1}-\mathbf{R}_{j}\right|^{-2}\right) \mathbf{P}^{(\text {in })} .
\end{aligned}
$$

Let $W_{i}$ be the statistical weight of a "scalar" $i$ th photon that arrives at the point $\mathbf{R}_{\mathrm{D}}$. Then, summing over all $N_{\text {ph }}$ detected photons, we obtain the following expressions for the polarized and depolarized components (for short, we omit the index "out"):

$$
\begin{gathered}
I_{\mathrm{pol}}=I_{X X}=\sum_{i=1}^{N_{\mathrm{ph}}} W_{i} P_{i x}^{2} \Gamma_{R}^{n_{i}}, \\
I_{\mathrm{depol}}=I_{Y X}=\sum_{i=1}^{N_{\mathrm{ph}}} W_{i} P_{i y}^{2} \Gamma_{R}^{n_{i}} .
\end{gathered}
$$

In the case of backward scattering, there is no $z$ component. Note that these formulas describe the noncoherent contribution of ladder diagrams, $I_{\beta \alpha}=$ $C_{\beta \beta \alpha \alpha}^{(L)}\left(0 \mid \mathbf{k}_{\mathrm{s}}, \mathbf{k}_{\mathrm{i}}\right)$.

In the case of an electromagnetic field, the polarization vector strongly fluctuates even for very large (on the order of $10^{5}$ ) statistical sample sizes. In [24], the authors analyzed the rate of depolarization as a function of the number $n$ of scattering events in the diffusive approximation. According to [24], in the case of isotropic single-scattering cross section, the residual depolarization after $n$ scattering events is given by

$$
P(n)=\frac{I_{\mathrm{pol}}(n)-I_{\mathrm{depol}}(n)}{I_{\mathrm{pol}}(n)+I_{\mathrm{depol}}(n)}=\frac{3(0.7)^{n-1}}{2+(0.7)^{n-1}} .
$$

Figure 4 represents the calculated function $P(n)$. Note that the number of scattering events is actually proportional to the length of the optical path; since the length of the path is proportional to the flight time, the function $P(n)$ illustrates the spreading of a light impulse in a strongly inhomogeneous medium. One can see that the depolarization indeed decays exponentially as the optical path increases; however, the decay rate differs 
from that predicted by the diffusive approximation. As the anisotropy increases, the characteristic length of depolarization increases, because, for large values of $\overline{\cos \theta}$, a photon must experience $(1-\overline{\cos \theta})^{-1}$ times greater number of collisions compared with the isotropic case to appreciably change its direction and, hence, the polarization. For $n>30$, the results are quite unstable. This is associated with the fact that the products of an odd number of components of the polarization vector directed along the boundary of the medium are equal to zero theoretically, whereas the stochastic result strongly fluctuates.

The calculations are performed as follows. For trajectories with a number of scattering events less than a certain number $n_{0}$, which varied from 20 to 50 , the calculation was performed by the above-described scheme. We assumed that, for $n>n_{0}$, the polarized and depolarized components are equal and, hence, can be calculated as half the intensity calculated for a scalar field:

$$
I_{\mathrm{pol}}(n)=I_{\mathrm{depol}}(n)=\frac{1}{2} I_{\text {scalar }}(n) .
$$

To control the process, we compared the results of simulation in the case of point scatterers with the known exact theoretical results.

In the case of Rayleigh scattering for the normal incidence and scattering at an angle of $180^{\circ}$, the exact solution [21,22] obtained within a generalization of the Milne solution for an electromagnetic field yields a value of $I_{\mathrm{pol}} / I_{\mathrm{depol}} \approx 1.92$ for the ratio of polarized to depolarized components of the noncoherent component. The numerical simulation yields $I_{\text {pol }} I_{\text {depol }} \approx 1.94$. The known value of the ratio of the polarized component of scattered radiation to the depolarized component allows one to determine the residual polarization of the noncoherent component of backscattering. The generalized Milne solution yields [19, 21, 22]

$$
\frac{I_{\mathrm{pol}}-I_{\mathrm{depol}}}{I_{\mathrm{pol}}+I_{\mathrm{depol}}} \approx 0.31 \text {, }
$$

while numerical simulation yields 0.326 . A close value of 0.33 was obtained in [23].

Let us define the height of the peak of the polarized component of coherent backscattering as

$$
h_{\mathrm{pol}}^{\mathrm{CBS}}=\frac{2 I_{\mathrm{pol}}-I_{\text {single }}}{I_{\mathrm{pol}}} .
$$

The theoretical value $[19,21,22]$ is $h_{\mathrm{pol}}^{\mathrm{CBS}} \approx 1.75$. The analysis of simulation data obtained in [2] yields a value of $h_{\mathrm{pol}}^{\mathrm{CBS}} \approx 1.4$, which is far from the theoretically predicted result. In [29], the authors obtained a value of

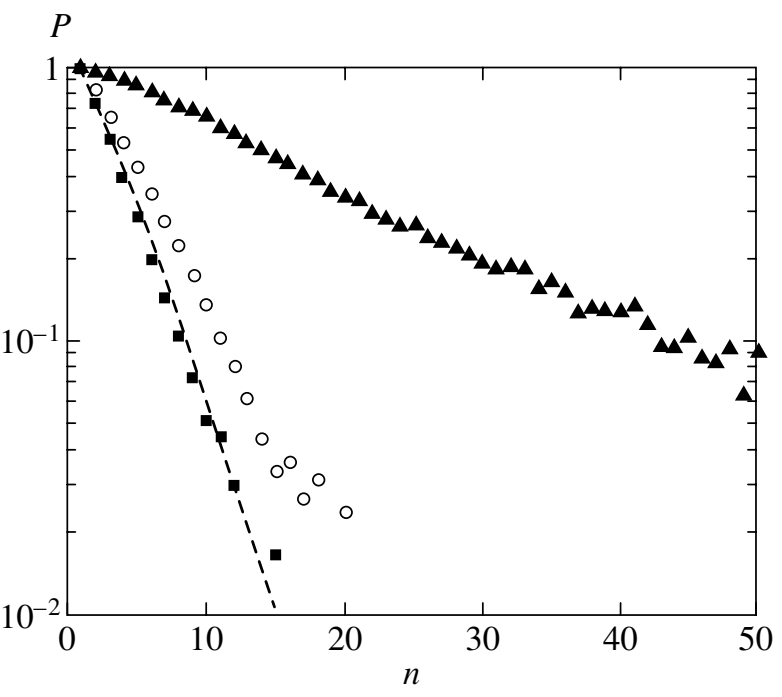

Fig. 4. Depolarization degree $P$ as a function of the number of scattering events $n$ for $\overline{\cos \theta}=0(\mathbf{\square}), 0.5(\bigcirc)$, and $0.9(\mathbf{\Lambda})$. A semi-infinite layer. The straight line represents the diffusive approximation.

$h_{\mathrm{pol}}^{\mathrm{CBS}} \approx 1.69$. The value $h_{\mathrm{pol}}^{\mathrm{CBS}} \approx 1.746$ calculated by us much better agrees with theory.

We calculated the time correlation function for the polarized and depolarized components of backscattered light by numerical simulation.

To calculate the time correlation functions of an electromagnetic field, we used the following formulas:

$$
\begin{gathered}
g_{\mathrm{pol}}^{(1)}(t)=\sum_{i=1}^{N_{\mathrm{ph}}} W_{i} P_{i x}^{2} \Gamma_{\mathrm{R}}^{n_{i}} \\
\times \exp \left(-2 \frac{t}{\tau} n_{i}\left(1-\frac{1}{n_{i}} \sum_{j}^{n_{i}} \cos \theta_{j}\right)\right), \\
g_{\text {depol }}^{(1)}(t)=\sum_{i=1}^{N_{\mathrm{ph}}} W_{i} P_{i y}^{2} \Gamma_{\mathrm{R}}^{n_{i}} \\
\times \exp \left(-2 \frac{t}{\tau} n_{i}\left(1-\frac{1}{n_{i}} \sum_{j}^{n_{i}} \cos \theta_{j}\right)\right),
\end{gathered}
$$

where $P_{i \alpha}$ is the polarization vector of the $i$ th photon with polarization $\alpha$ that arises under the action of a sequence of $n_{i}$ tensor operators of the form (4.2) and $\theta_{j}$ is the scattering angle of the $j$ th scattering event.

Figure 5 represents the results of simulation of the time correlation function of field for the polarized and depolarized components for the Rayleigh scattering, $\overline{\cos \theta}=0$, and for the case of a strongly anisotropic sys- 


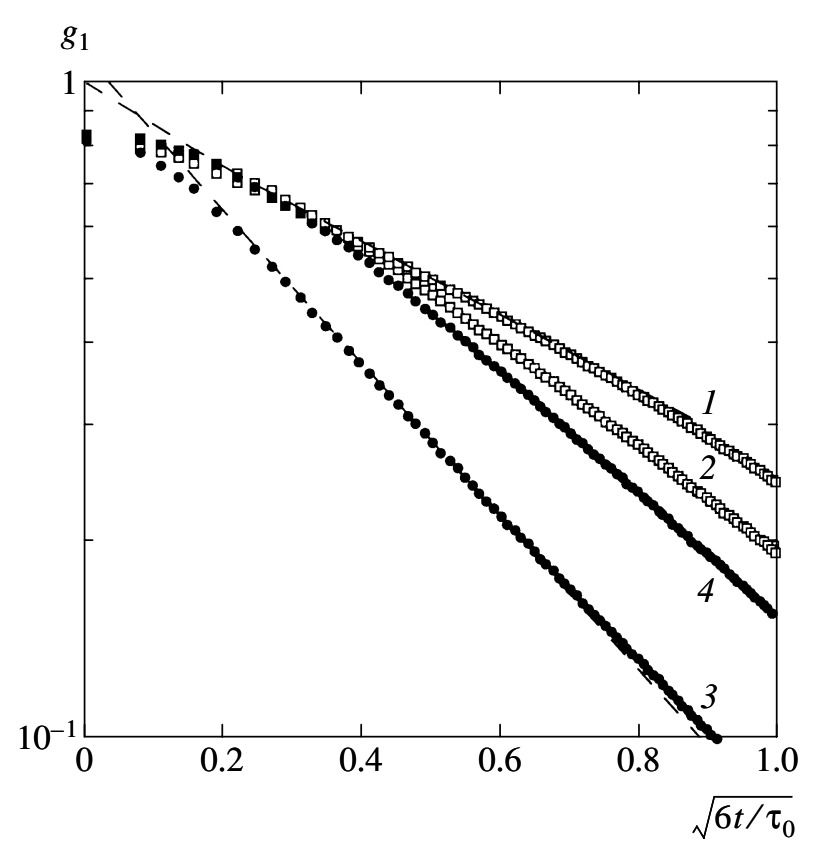

Fig.5. The time correlation function of a scattered field: $g_{X X}^{(1)}$ $(\square), g_{X Y}^{(1)}(\bullet) ; 1$ and 3 - medium with the isotropic scattering, $\overline{\cos \theta}=0 ; 2$ and $4-$ anisotropic, $\overline{\cos \theta}=0.9$. The dashed lines show the slops $\gamma_{\text {pol }} \approx 1.42$ and $\gamma_{\text {depol }} \approx 2.68$ for isotropic case.

tem with the indicatrix stretched forward, $\overline{\cos \theta}=0.9$. One can see that, in the case of linearly polarized light, the decay rate of the time correlation function in the units of $\tau$ appreciably depends on the anisotropy parameter $\overline{\cos \theta}$, in contrast to nonpolarized light.

The diffusive character of light propagation in the multiple scattering regime is responsible for the linear decay of the field time correlation function of the form [15]

$$
g^{(1)}(t) \sim 1-\gamma \sqrt{6 t / \tau}
$$

where $\gamma$ is the slope ratio that determines the decay rate of the time correlation function. Note that, according to (3.5), the initial small parameter in time is $(t / \tau)(1-$ $\overline{\cos \theta})$. In the case of a strongly anisotropic scattering cross section, this quantity remains small for sufficiently large values of the parameter $t / \tau$ for which the dependence of $g^{(1)}(t)$ on $\sqrt{t / \tau}$ may strongly differ from a linear function.

In the case of Rayleigh scattering, the theory predicts $[19,22]$ the following slope ratios for the polarized and depolarized components: $\gamma_{\text {pol }} \approx 1.44$ and $\gamma_{\text {depol }} \approx 2.75$. The analysis within the diffusive approximation yields [16] $\gamma_{\text {pol }} \approx 1.6$ and $\gamma_{\text {depol }} \approx 2.7$, which are close to the experimental data $\gamma_{\text {pol }} \approx 1.6 \pm 0.1$ and $\gamma_{\text {depol }} \approx 2.8 \pm 0.2$. These experimental data were obtained for a suspension of latex particles with a diameter of $D=0.091 \mu \mathrm{m}$, which is much less than the wave- length; i.e., the scattering is close to the Rayleigh scattering.

Note that, just as in a real experiment [15], one cannot eliminate the nonlinear region for very small values of time, which is attributed to the finiteness of the aperture and the finiteness of the number of scattering events considered; the theoretically predicted linear region with the slope ratios $\gamma_{\mathrm{pol}} \approx 1.42$ and $\gamma_{\mathrm{depol}} \approx 2.68$ starts at $\sqrt{t / \tau}=0.15$. As the anisotropy parameter increases, the decay rates of the polarized and depolarized components approach each other. The sum of the polarized and depolarized components, i.e., the time correlation function of nonpolarized light is described by a curve close to the curve obtained during the simulation of the time correlation function of a scalar field.

\section{CONCLUSION}

In the theory of coherent and correlation phenomena of multiple scattering, both the cyclic diagrams that describe the interference component of backscattering $[33,24]$ and the diagrams that describe the field correlations [34] can be reduced to ladder diagrams. This allows one to describe the above phenomena within the framework of the relevant Bethe-Salpeter equation. The formal difference from the original ladder diagrams that describe the transfer of the main, noncoherent, component of scattered radiation consists in the introduction of additional coefficients to the vertices of ladder diagrams. These coefficients describe the phase shift between the fields that appear in the definition of the propagator of the Bethe-Salpeter equation. In the present paper, by juxtaposing the representation of the Bethe-Salpeter equation as a ladder diagram series with the modeling of random trajectories, we have shown that the consideration of phase relations in stochastic modeling also reduces to the addition of appropriate coefficients in each scattering event experienced by a photon as it moves along a random trajectory.

The semianalytic Monte Carlo method developed allows one to compare numerical results with theoretical predictions at each step of simulation. The possibility of such a comparison allows one to significantly reduce the simulation time by using analytic results instead of numerical ones at large distances between the input and output points of radiation, where theoretical predictions are certainly correct.

The analysis carried out has shown that scattering is indeed of multiple character only in the case of a nonabsorbing semi-infinite medium. In all other cases, each scattering event gives rise to coefficients of the form $D_{\mathrm{s}} \overline{q^{2}} t=2(t / \tau)\left(l / l^{*}\right)$ when simulating the time correlation functions with diffusive decay of fluctuations or $k l \sin \theta_{\mathrm{s}}$ for coherent backscattering; these coefficients lead to a rapid decay of a wave packet. The decay rate of coherent phenomena is determined by the parameters $(t / \tau)$ and $k l^{*} \sin \theta_{\mathrm{s}}$, which may be substan- 
tially greater than the original parameters $(t / \tau)\left(l / l^{*}\right)$ and $k l \sin \theta_{\mathrm{s}}$ in the case of a strongly anisotropic phase function, $1-\overline{\cos \theta_{\mathrm{s}}} \ll 1$.

The simulation error is primarily determined by the sample size, i.e., by the number $N_{\mathrm{ph}}$ of emitted photons and by the maximal number $n^{(\max )}$ of the scattering events taken into consideration. We continued to follow up the trajectory of a photon up to $10^{4}$ scattering events. In this case, the results become stable to within a percent even for about 1000 emitted photons; for $N_{\mathrm{ph}} \sim 10^{5}$, the relative error in the scattering intensity is on the order of $10^{-4}$. However, in view of the diffusive character of the propagation of radiation in an unbounded medium, the contributions of the scattering events of extremely high orders still continue to contribute when one simulates the intensity. Therefore, the statistics of photons with high-order scattering proves to be insufficient at large distances from the input point, and the result depends on the choice of the number $n^{(\max )}$. Comparing, in the isotropic case, numerical results with the theoretical results that follow from the Milne solution and its generalizations, we have obtained that the above-mentioned restriction on the number of scattering events gives an understated result for the intensity of scattered radiation: on the order of $2 \%$ for a scalar field and on the order of 5\% for an electromagnetic field, even for sample sizes of up to several millions. It is this circumstance why we complement stochastic modeling with analytic calculations, applying analytic calculations within the diffusive approximation instead of modeling in the case of large distances between the input and output points (or when the number of scattering events exceeds a certain fixed number); the accuracy of these analytic calculations are easily controlled by increasing the above-mention fixed parameter.

In the case of modeling the time correlation function or the peak of coherent backscattering for the parameters $(t / \tau)\left(l / l^{*}\right)$ and $k l^{*} \sin \theta_{\mathrm{s}}$ on the order of 0.01 and greater, an additional contribution (combined with the use of analytic calculations) of scattering events of extremely high orders virtually does not influence the result. In the range of large values of $(t / \tau)\left(l / l^{*}\right)$ and $k l^{*} \sin \theta_{\mathrm{s}}$ on the order of unity, the relative contribution increases again; however, in this range of parameters, the very physical model and, in particular, the ladder approximation cease to be valid.

The description of multiple scattering, including coherent phenomena, in terms of ladder diagram series is valid up to the parameter $\lambda / l$. Thus, when the time correlation function decreases as time increases or the peak of coherent backscattering decreases as the scattering angle increases by a factor greater than $l / \lambda$, the phenomena described here cease to be observable in a real experiment against the background of the contributions of "nonladder" diagrams that take into account the phase shifts of fields during multiple rescattering processes.
Usually, multiple scattering, including coherent phenomena, is described in terms of a scalar field. Our results show that, for the backward scattering, the consideration of the electromagnetic nature of light leads to an essentially different quantitative description compared with that in the case of a scalar field because a considerable part of backward scattered radiation consists of low-order contributions. For example, the decay rate of the time correlation function of the polarized component is much less, while that of the depolarized component is much greater, than the decay rate in the case of nonpolarized light; the latter decay rate virtually coincides with that in the scalar case. The polarized component of backscattered light is almost twice as large as the depolarized component.

The method of numerical simulation developed allows one to judge the number of scattering events experienced by light transmitted through a layer of strongly inhomogeneous opaque medium by the value of residual polarization. This information is additional to that obtained from the measurements of attenuation of nonpolarized light, from which one derives the transport mean free path. The comparative analysis carried out allows one to significantly simplify the simulation of radiative transfer and coherent phenomena in randomly inhomogeneous strongly scattering media, such as liquid crystals, biological tissues, etc., and to significantly extend the application domain of these methods.

The results obtained allow one to extend the applicability domain of the methods based on the coherent and correlation properties of diffusely scattered light.

\section{ACKNOWLEDGMENTS}

This work was supported by the Russian Foundation for Basic Research (project no. 02-02-16577), the Royal Society (project no. 15298), and NATO (project no. PST.CLG.979652).

\section{REFERENCES}

1. M. Ospeck and S. Fraden, Phys. Rev. E 49, 4578 (1994).

2. T. Iwai, H. Furukawa, and T. Asakura, Opt. Rev. 2, 413 (1995).

3. K. Ishii, T. Iwai, and T. Asakura, Opt. Rev. 4, 643 (1997).

4. S. E. Skipetrov and S. S. Chesnokov, Kvantovaya Élektron. (Moscow) 25, 753 (1998).

5. R. Lenke and G. Maret, Eur. Phys. J. B 17, 171 (2000).

6. S. E. Skipetrov and I. V. Meglinskiǔ, Zh. Éksp. Teor. Fiz. 113, 1213 (1998) [JETP 86, 661 (1998)].

7. R. Lenke, R. Tweer, and G. Maret, J. Opt. A: Pure Appl. Opt. 4, 293 (2002).

8. D. A. Zimnyakov, Yu. P. Sinichkin, I. V. Kiseleva, and D. N. Agafonov, Opt. Spektrosk. 92, 831 (2002) [Opt. Spectrosc. 92, 765 (2002)].

9. V. L. Kuz'min and I. V. Meglinskiŭ, Pis'ma Zh. Éksp. Teor. Fiz. 79, 139 (2004) [JETP Lett. 79, 109 (2004)]. 
10. V. L. Kuz'min and I. V. Meglinskiı̆, Opt. Spektrosk. 97, 108 (2004) [Opt. Spectrosc. 97, 100 (2004)].

11. B. A. van Tiggelen and S. E. Skipetrov, Wave Scattering in Complex Media: From Theory to Applications (Kluwer Academic, Dodrecht, 2003).

12. M. C. W. van Rossum and Th. N. Nieuwenhuizen, Rev. Mod. Phys. 71, 313 (1999).

13. I. M. Sobol', The Monte Carlo Method (Nauka, Moscow, 1985) [in Russian].

14. P. E. Wolf and G. Maret, Phys. Rev. Lett. 55, 2696 (1985).

15. D. J. Pine, D. A. Weitz, P. M. Chaikin, and E. Herbolzheimer, Phys. Rev. Lett. 60, 1134 (1988).

16. F. C. MacKintosh and S. John, Phys. Rev. B 40, 2383 (1989).

17. M. I. Mishchenko, Phys. Rev. B 44, 12597 (1991).

18. M. I. Mishchenko, J. Quant. Spectrosc. Radiat. Transf. 56, 673 (1996).

19. E. Amic, J. M. Luck, and T. M. Nieuwenhuizen, J. Phys. I 7, 445 (1997).

20. M. I. Mishchenko, J. M. Luck, and T. M. Nieuwenhuizen, J. Opt. Soc. Am. A 17, 888 (2000).

21. V. L. Kuz'min, Opt. Spektrosk. 93, 482 (2002) [Opt. Spectrosc. 93, 439 (2002)].

22. V. L. Kuz'min and E. V. Aksenova, Zh. Éksp. Teor. Fiz. 123, 929 (2003) [JETP 96, 816 (2003)].

23. L. F. Rojas-Ochoa, D. Lacoste, R. Lenke, et al., J. Opt. Soc. Am. A 21, 1799 (2004).
24. E. Akkermans, P. E. Wolf, R. Maynard, et al., J. Phys. (Paris) 49, 77 (1988).

25. Yu. N. Barabanenkov and V. D. Ozrin, Zh. Éksp. Teor. Fiz. 94 (6), 56 (1988) [Sov. Phys. JETP 67, 1117 (1988)].

26. I. V. Meglinskiı̌ and S. J. Matcher, Opt. Spektrosk. 91, 692 (2001) [Opt. Spectrosc. 91, 654 (2001)].

27. D. Y. Churmakov, I. V. Meglinski, and D. A. Greenhalgh, Phys. Med. Biol. 47, 4271 (2002).

28. A. Ishimaru, Wave Propagation and Scattering in Random Media (Academic, New York, 1978; Mir, Moscow, 1981).

29. R. Lenke and G. Maret, Eur. Phys. J. B 17, 171 (2000).

30. E. Tinet, S. Avrillier, and J. M. Tualle, J. Opt. Soc. Am. A 13, 1903 (1996).

31. G. I. Marchuk, G. A. Mikhailov, M. A. Nazaraliev, R. A. Darbinjan, B. A. Kargin, and B. S. Elepov, The Monte-Carlo Methods in Atmospheric Optics (Springer, Berlin, 1980).

32. G. Maret and P. E. Wolf, Physica B (Amsterdam) 65, 409 (1987).

33. A. Golubentsev, Zh. Éksp. Teor. Fiz. 86, 47 (1984) [Sov. Phys. JETP 59, 26 (1984)].

34. M. J. Stephen, Phys. Rev. B 34, 7564 (1986). 